\title{
Nuevas competencias digitales en estudiantes potenciadas con el uso de Realidad Aumentada. Estudio Piloto
}

\section{(New digital skills in students empowered with the use of Augmented Reality. Pilot Study)}

\author{
Inés María González Vidal \\ Beatriz Cebreiro López \\ Lorena Casal Otero \\ Universidad Santiago de Compostela (España)
}

DOI: http://dx.doi.org/10.5944/ried.24.1.27501

\section{Cómo referenciar este artículo:}

Vidal, I. M. G., Cebreiro López, B., y Casal Otero, L. (2021). Nuevas competencias digitales en estudiantes potenciadas con el uso de Realidad Aumentada. Estudio Piloto. RIED. Revista Iberoamericana de Educación a Distancia, 24(1), pp. 137157. doi: http://dx.doi.org/10.5944/ried.24.1.27501

\section{Resumen}

En la actualidad, las tecnologías aplicadas a la Educación se colocan en una dimensión superior en el contexto educativo, en consecuencia, surge la necesidad de visualizar nuevas competencias digitales para integrar el conocimiento tecnológico de los medios con el conocimiento didáctico de la utilización de los mismos. El objetivo de este trabajo es potenciar nuevas competencias digitales con el uso de la Realidad Aumentada (RA) en estudiantes universitarios de 4to año de la carrera de Pedagogía. El diseño metodológico está basado en un estudio piloto con enfoque mixto, se trabaja con un grupo de 9 estudiantes en la asignatura Formación en Red, se aplican cuestionarios y se usa estadística descriptiva (prueba Chi cuadrado). Las variables analizadas son la motivación, las expectativas de uso de la RA, el éxito de la tarea y la satisfacción al concluir la actividad. El análisis didáctico identifica segmentos vinculados a fases no clásicas en este tipo de análisis (espacio-tiempo, contenido-método, discursos, interacción y ambiente). La experiencia contribuye a la preparación, diseño, producción y utilización de tecnologías avanzadas en la educación; también se contempla al estudiante en un nuevo rol como prosumidor de contenidos virtuales, lo que constituye una nueva competencia digital antes reservada a desarrolladores informáticos.

Palabras clave: realidad aumentada; enseñanza superior; estudiantes universitarios; competencias digitales; entornos digitales; tecnología educativa. 


\begin{abstract}
Currently, the technologies applied to Education are placed in a higher dimension in the educational context, consequently, the need arises to visualize new digital competences to integrate technological knowledge of the media with didactic knowledge of their use. At this work we aim at promoting new digital competences with the use of Augmented Reality (AR) in 4th year university students of the Pedagogy degree. The methodological design is based on a pilot study with a mixed approach, we work with a group of 9 students in the subject called Network Training, questionnaires are applied and descriptive statistics are used (Chi square test). The variables analyzed are motivation, expectations of use of AR, task success and satisfaction at the end of the activity. The didactic analysis identifies segments linked to nonclassical phases in this type of analysis (space-time, content-method, discourses, interaction and environment). The experience contributes to the preparation, design, production and use of advanced technologies in education; The student is also seen in a new role as a prosumer of virtual content, which constitutes a new digital competence previously reserved for computer developers.
\end{abstract}

Keywords: augmented reality; higher education; university students; digital skills; digital environments; educational technology.

En las últimas décadas, ha ganado relevancia la investigación sobre la integración de las nuevas Tecnologías de la Información y las Comunicaciones (TIC) en la educación (San Nicolás, Vargas y Moreira, 2012). Las nuevas tecnologías aplicadas a la Educación se colocan en una dimensión superior como único recurso que provee de oportunidades inigualables a los procesos educativos para permitir la continuación del curso escolar de manera online (Fainholc, 2008; García-Ruiz, Aguaded y Bartolomé, 2017). Se ha producido un cambio cultural y tecnológico, que transforma rápidamente la sociedad y por consiguiente los procesos educativos (Castañeda Quintero y Adell, 2013). Al mismo tiempo, surgen nuevas competencias digitales cotidianas que contribuyen a la integración de las TIC en la Educación (Fernández, Fernández y Cebreiro, 2016).

Las instituciones educativas potencian nuevas tendencias innovadoras para la puesta en marcha de métodos y estilos de aprendizaje que aprovechen al máximo los recursos materiales, tecnológicos y humanos existentes (García-Ruiz, Aguaded y Bartolomé, 2017).

Algunos autores visualizan las carencias formativas existentes respecto a las nuevas competencias digitales que se exigen en la sociedad actual (Marzal, 2008; Palomeque-Córdova, 2020). La necesidad de una alfabetización tecnológica constante para formar estudiantes inteligentes, críticos de las tecnologías avanzadas es un requisito indispensable para el desarrollo profesional de la nueva generación (Aguaded Gómez, Marín Gutiérrez y Díaz Pareja, 2015). 
También se percibe la importancia de realizar modificaciones curriculares con el fin de incorporar el tratamiento de la información y la formación en tecnologías avanzadas que deben poseer los estudiantes y profesores, respecto al conocimiento de los medios audiovisuales, informáticos y la integración de las TIC en el proceso educativo (Labra, 2010). Algunos aspectos que pueden limitar el uso de tecnologías avanzadas en las prácticas educativas se relacionan con los actores del proceso, al no estar preparado para el diseño y la producción de medios, así como para el manejo técnico y su uso didáctico (Ramírez García y González Fernández, 2016). En este sentido, qué acciones se pueden desenvolver para que trascienda el carácter instrumental de los medios y lograr integrar el conocimiento tecnológico avanzado con el conocimiento didáctico de su utilización (Ramírez García y González Fernández, 2016).

En este contexto, el objetivo de este trabajo es potenciar nuevas competencias digitales con el uso de la Realidad Aumentada (RA) en estudiantes de 4to año de la carrera de Pedagogía. Para ello será necesario: 1) analizar la literatura científica e identificar experiencias didácticas similares que utilicen RA en diversas áreas de conocimiento; 2) desarrollar acciones formativas encaminadas a familiarizar a los estudiantes con la tecnología de RA para fomentar el desarrollo de aplicaciones futuras; 3) por último hacer una valoración de los resultados de la experiencia didáctica.

La experiencia didáctica con el uso de dispositivos móviles y RA contribuyó a visualizar al estudiante como prosumidores de contenidos virtuales aplicados a la educación. Además, se vislumbra la necesidad de promover acciones formativas sistemáticas que contribuyan a la preparación de los discentes en el diseño, la producción y la utilización de los recursos virtuales que faciliten en el futuro el uso de tecnologías avanzadas en los centros educativos.

\section{La tecnología de Realidad Aumentada en la educación}

La utilización de recursos tecnológicos en entornos escolares y el acceso a contenidos educativos digitales a través de dispositivos móviles inteligentes es cada vez más frecuente (Montoya, 2009). Es una realidad también el aumento de la brecha digital en aquellos colectivos que no tienen acceso a las mismas tecnologías o no poseen las capacidades suficientes para trabajar con ellas (García Aretio, 2017). En este sentido, son necesarias un mayor número de investigaciones y mejores políticas de gobiernos que garanticen la armonía entre equidad e innovación en la educación (Vila Merino, 2012).

El uso de dispositivos móviles inteligentes adquiere gran connotación en la educación (De la Horra Villacé, 2017), debido a la reducción de costes en equipamientos electrónicos y lo versátil que resultan estos dispositivos (Barroso Osuna y Cabero Almenara, 2016). Este hecho influye en la deslocalización de las tecnologías y el acceso a la información, se minimizan las barreras espacio temporal 
propias de la comunicación interpersonal (Cabero Almenara, Fernández Robles y Marín Díaz, 2017).

En este escenario, aparecen las aplicaciones de la RA que permiten al usuario ver el mundo real con objetos virtuales superpuestos o compuestos con el mundo real (Bogen, Wind y Giuliano, 2006). Los objetos reales y virtuales coexisten en el mismo espacio y esta escena se puede observar a través de una pantalla de video convencional (Arribas, Gutiérrez, Gil y Santos, 2014; Noh, Sunar y Pan, 2009).

La RA permite la exploración no planificada y el descubrimiento de la naturaleza (Johnson, Levine, Smith y Stone, 2010), es decir la RA complementa la "Realidad", en lugar de reemplazarla por completo como lo hace la Realidad Virtual (RV) (Azuma, 1997). En este sentido, la RV y la RA forman parte de una línea continua donde en uno de los extremos se encuentra la "Realidad" y del otro lado de la línea se encuentra la RV (Escartín, 2000). Entre ambas concepciones se sitúa la RA, la misma requiere de la manipulación o la visualización de objetos que no están al alcance de todos, bien por su naturaleza abstracta, científica o espacial (Milgram, Takemura, Utsumi y Kishino, 1995).

En la educación, la RA presupone un conjunto de tecnologías que permiten al estudiante visualizar contenidos educativos a través de un dispositivo móvil inteligente (Marín-Díaz y Sampedro-Requena, 2020). Las destrezas adquiridas por los estudiantes a través de experiencias con RA presuponen habilidades para el acceso, análisis, evaluación y producción creativa de nuevos conocimientos (Cabero Almenara y García, 2016).

La RA es una tecnología avanzada con muchas posibilidades de aplicación en el terreno educativo, ayuda a aumentar la motivación en los estudiantes, contribuye a disminuir el tiempo necesario para comprender temas complejos, permite recrear ambientes difíciles de reproducir y facilita la interacción entre objetos y recursos de diversas naturalezas (Wu, Lee, Chang y Liang, 2013). La RA posibilita un aprendizaje interactivo, atractivo y motivador para estudiantes y profesores (Deshpande, Kank, Armanyous, Singh y Kalita, 2020).

No obstante, es necesario un plan de acción apropiado y posibles soluciones para poder aplicar con éxito la RA (Klopfer y Squire, 2008). Una de las limitaciones señaladas en la literatura se refiere a los entornos de aprendizaje con RA caracterizados por sobrecargas cognitivas, debido a la gran cantidad de información contenida, a los múltiples dispositivos tecnológicos y a las complejas tareas a desarrollar (Klopfer, 2008). La RA puede crear confusión en los estudiantes, que pierden de vista dónde termina el juego y dónde comienza la realidad, lo que podría constituir una amenaza para la seguridad física de los estudiantes (Dunleavy, Dede y Mitchell, 2009). Otros autores consideran que, aunque la RA es una tecnología que ayuda y facilita la comprensión de los contenidos curriculares, son necesarios recursos digitales y centros de capacitación para familiarizarse con esta tecnología (Marín-Díaz y Sampedro-Requena, 2020). 
Para evidenciar la importancia y diversidad de las aplicaciones con RA existentes en el contexto educativo, se identifican algunas experiencias didácticas en diversos dominios:

1. Aplicación para experiencia didáctica en museos (Museo de Juguetes en Nápoles). En los museos por su propia naturaleza, y por el hecho de preservar y proteger el objeto, se limita el disfrute del visitante a la observación del objeto expuesto. No obstante, los niños prefieren realizar esta experiencia de aprendizaje interactuando con el objeto en cuestión. Entonces ¿Cómo dar vida a los objetos almacenados en espacios como los museos? La respuesta es dada con la RA, esta tecnología permite al juguete, que sólo es accesible visualmente, revivir emociones con ayuda de la RV (Sarracino, 2014).

2. Aplicación para la difusión didáctica del patrimonio. El empleo de la RV y RA para reconstruir escenarios en $3 \mathrm{D}$ de distintos periodos históricos permite interactuar con castillos, torres de época islámica, entre otros escenarios. Con esta tecnología, se integran conceptos y técnicas innovadoras, muy útiles en especialidades como la arqueología y la restauración (Usó y García, 2014).

3. Aplicación en Juegos didácticos con RA. Pompeya es un juego virtual donde los personajes virtuales se superponen en un entorno real. Los resultados de esta experiencia con RA fue la reconstrucción del patrimonio cultural en formato digital. Con el desarrollo de trabajos de este tipo se preserva, protege e interpreta la cultura e historia; también se puede mejorar y estimular la comprensión del estudiante (Noh, Sunar y Pan, 2009; El-Hakim, Beraldin, Picard y Godin, 2004).

4. Aplicaciones on-line con RA. Una experiencia didáctica interesante con RA, es el diseño de un sistema tele-robótico online donde un robot es controlado por Internet, el sistema es considerado con autonomía ajustable. Con ayuda de RA se predicen las acciones del robot. El uso extensivo de la Web con RA permite un fácil acceso sin restricciones espacio-temporal y abre la posibilidad de la enseñanza a distancia (Marín, Sanz y Del Pobil, 2003).

5. Aplicación en laboratorios. En el campo de la ingeniería, los trabajos en los laboratorios son muy importantes para confirmar las teorías estudiadas. En este caso, se desarrolló un nuevo entorno de aprendizaje a través de un manual de instrucción Web basado en contenidos e-Learning. La RA se utiliza para sincronizar el manual de instrucción Web y el experimento (Toguchi, Sasaki, Mizuno y Shikoda, 2011).

\section{MÉTODO}

El objetivo del trabajo es potenciar nuevas competencias digitales con el uso de la Realidad Aumentada (RA) en estudiantes de 4to año de la carrera de Pedagogía de la Facultad de Ciencias de la Educación de la Universidad Santiago de Compostela. El diseño metodológico está basado en un estudio piloto (Van Teijlingen y Hundley, 
2002) con enfoque mixto (análisis cualitativo y cuantitativo) además de ser un estudio exploratorio (Marshall y Rossman, 2014; Preissle, 2008). La muestra es un grupo reducido de 9 estudiantes de la asignatura Formación en Red (Tecnología Educativa).

Primero se realiza un análisis documental de la literatura científica y se identifican ejemplos de aplicaciones de RA en diversos contextos educativos. La selección de las herramientas de RA se realiza con la ayuda de expertos, atendiendo al grado de interacción deseada entre los actores. Desde el punto de vista didáctico la experiencia con RA se divide en etapa pretest donde se construye la propuesta didáctica a realizar, y la etapa postest corresponde a la ejecución.

Los instrumentos para la recolección de datos son los cuestionarios, y para describir y analizar las características del conjunto de datos se utiliza la estadística descriptiva. Se definen variables cualitativas ordinales (donde no es necesario que el intervalo entre mediciones sea uniforme): motivación, las expectativas de uso de la RA, el éxito de la tarea y la satisfacción al concluir la actividad. Basado en el comportamiento descriptivo de las variables expectativas de uso de la RA y el éxito de la tarea y el supuesto de que no existe relación entre ellas, se realiza una prueba estadística Chi cuadrado.

\section{Etapa pretest}

A los estudiantes de 4to año de la carrera de Pedagogía se les brinda una formación teórica y práctica necesaria para familiarizarse con los recursos tecnológicos educativos necesarios para el aprendizaje, la innovación y la adecuación de metodologías y recursos didácticos. Específicamente en la asignatura Formación en Red (Tecnología Educativa) se diseñan, gestionan y evalúan materiales digitales. Los estudiantes exploran diversos escenarios flexibles de enseñanza con tecnologías: plataformas de formación y colaboración, ámbitos personalizados de aprendizaje (Web Social), PLEs y comunidades virtuales de aprendizaje. En este sentido, los estudiantes deben desarrollar durante el semestre un trabajo de curso individual, que comienza con la identificación de un problema educativo, posteriormente deben hacer una propuesta de mejora de la práctica educativa utilizando para ello tecnologías emergentes aplicadas a la educación.

La etapa pretest establece la propuesta didáctica a realizar como un vínculo entre contenido-método a partir del cual se configura las clases. Siguiendo a Stodolsky (1991) es posible identificar diferentes segmentos o unidades de sentido claramente demarcados, a partir de los cuales es posible efectuar los procesos de análisis y reflexión. Los segmentos se vinculan a fases: espacio-tiempo, contenido-método, discursos, interacción y ambiente. Esta clasificación no es habitual en el análisis didáctico, no obstante, es considerada en este estudio como transversal del proceso educativo. Se diseñan un conjunto de actividades formativas para familiarizar al 
estudiante con herramientas de RA, y luego aplican los conocimientos adquiridos a sus proyectos individuales de curso.

\section{RESULTADOS}

\section{Consulta a expertos para la selección de las herramientas de RA}

En esta etapa se analizaron los recursos cuya complejidad y funcionalidad son apropiados para la experiencia didáctica que se pretende, para eso se realiza una consulta a expertos y desarrolladores de RA. El CESGA (Centro de Supercomputación de Galicia) es una institución que goza de gran importancia en el territorio, ya que contribuye al avance de la Ciencia y la Tecnología, mediante la investigación y aplicación de la computación y las comunicaciones, en beneficio de la Sociedad y en particular a la Educación. Expertos y desarrolladores de este centro, mostraron las tecnologías de RA más populares en el contexto escolar, y que están siendo utilizadas en centros del territorio gallego e internacional. En la figura 1 aparecen las herramientas RA seleccionadas, se presentan los parámetros y características analizadas para la elección. 
Figura 1. Características de las herramientas de RA seleccionadas

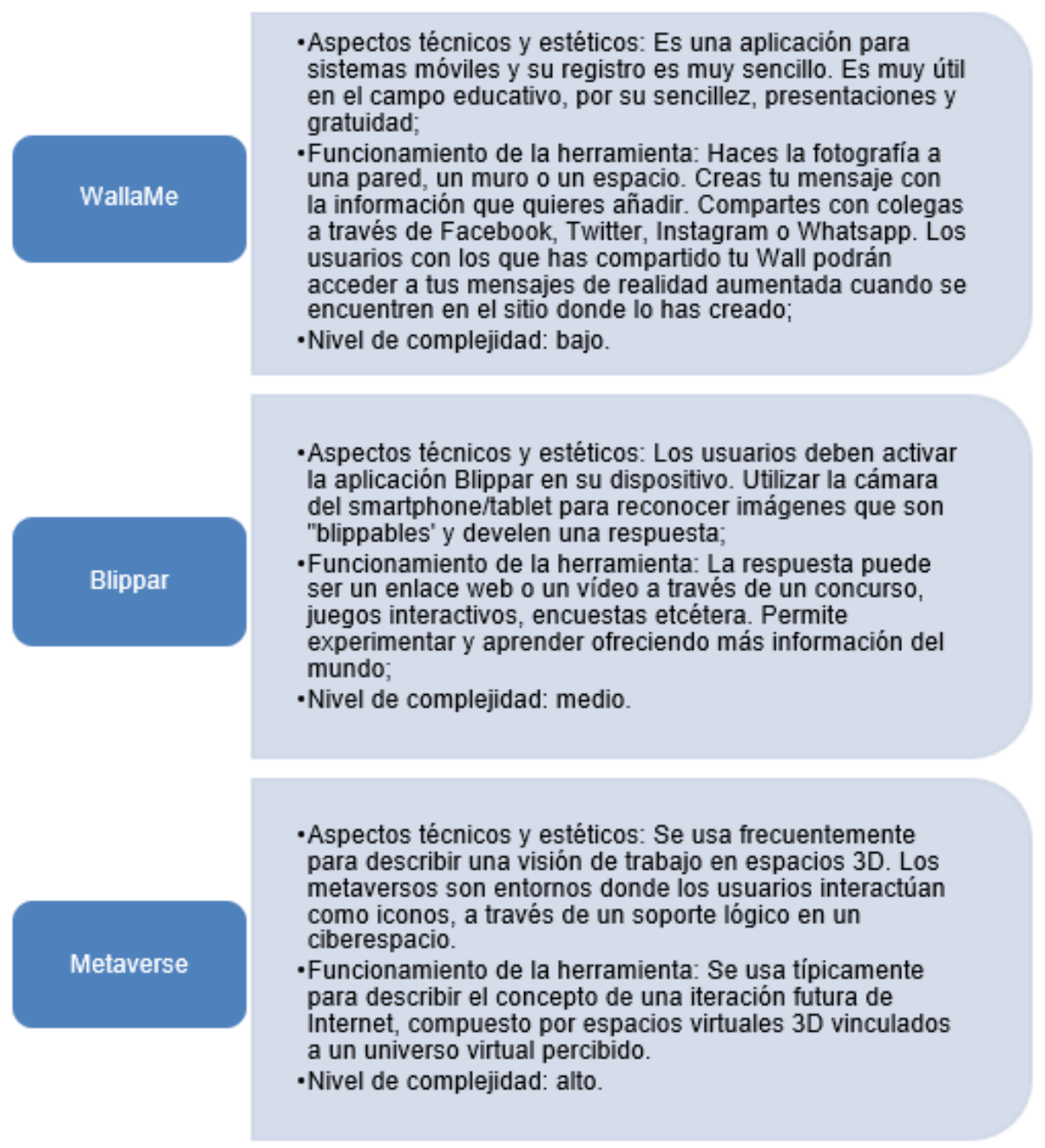

Fuente: elaboración propia utilizando LibreOffice.

\section{Etapa postest}

Esta etapa muestra la ejecución de la propuesta didáctica descrita en la etapa pretest. El proceso de análisis didáctico se realiza a través de los segmentos que son unidades de sentido que se vinculan a las fases: espacio-tiempo, contenido-método, discursos, interacción y ambiente. La experiencia didáctica con RA se divide en dos clases, primera clase tiene como objetivo familiarizar a los estudiantes con algunas 
herramientas de RA, presentar algunos ejemplos de aplicación en diversos dominios, $\mathrm{y}$ fomentar su uso de la RA en ambientes educativos especialmente en sus trabajos de curso, ver figura 2.

Figura 2. Primera clase de la experiencia didáctica con RA

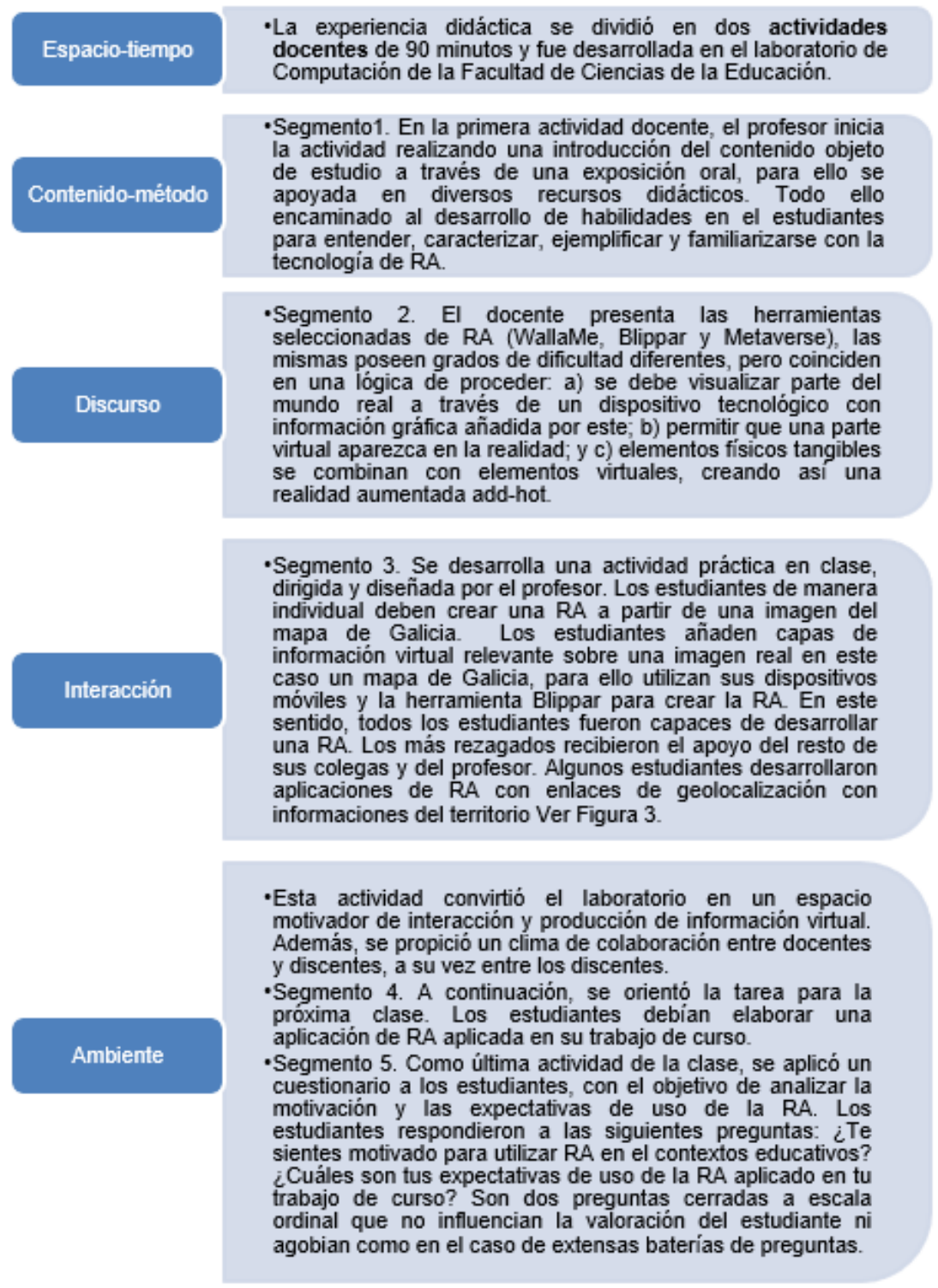

Fuente: elaboración propia utilizando LibreOffice. 
En la figura 3 se muestran ejemplos de aplicaciones de RA desarrolladas por los estudiantes en el laboratorio de computación de la facultad.

Figura 3. Ejemplos de RA desarrolladas por los estudiantes

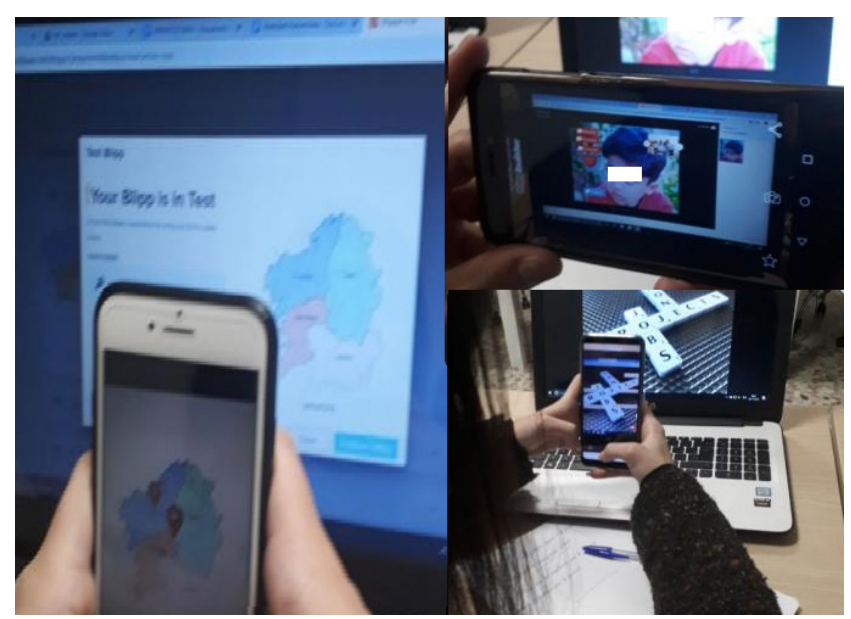

Fuente: elaboración propia a partir de fotos tomadas en clase.

La segunda clase es un taller, implica una metodología científica de trabajo que integra la teoría y la práctica, es una actividad esencial para desarrollar proyectos en general. El objetivo es que los estudiantes expongan sus aplicaciones de RA y fomentar el intercambio de experiencia entre todos los actores del proceso, ver figura 4. 
Figura 4. Segunda clase de la experiencia didáctica con RA

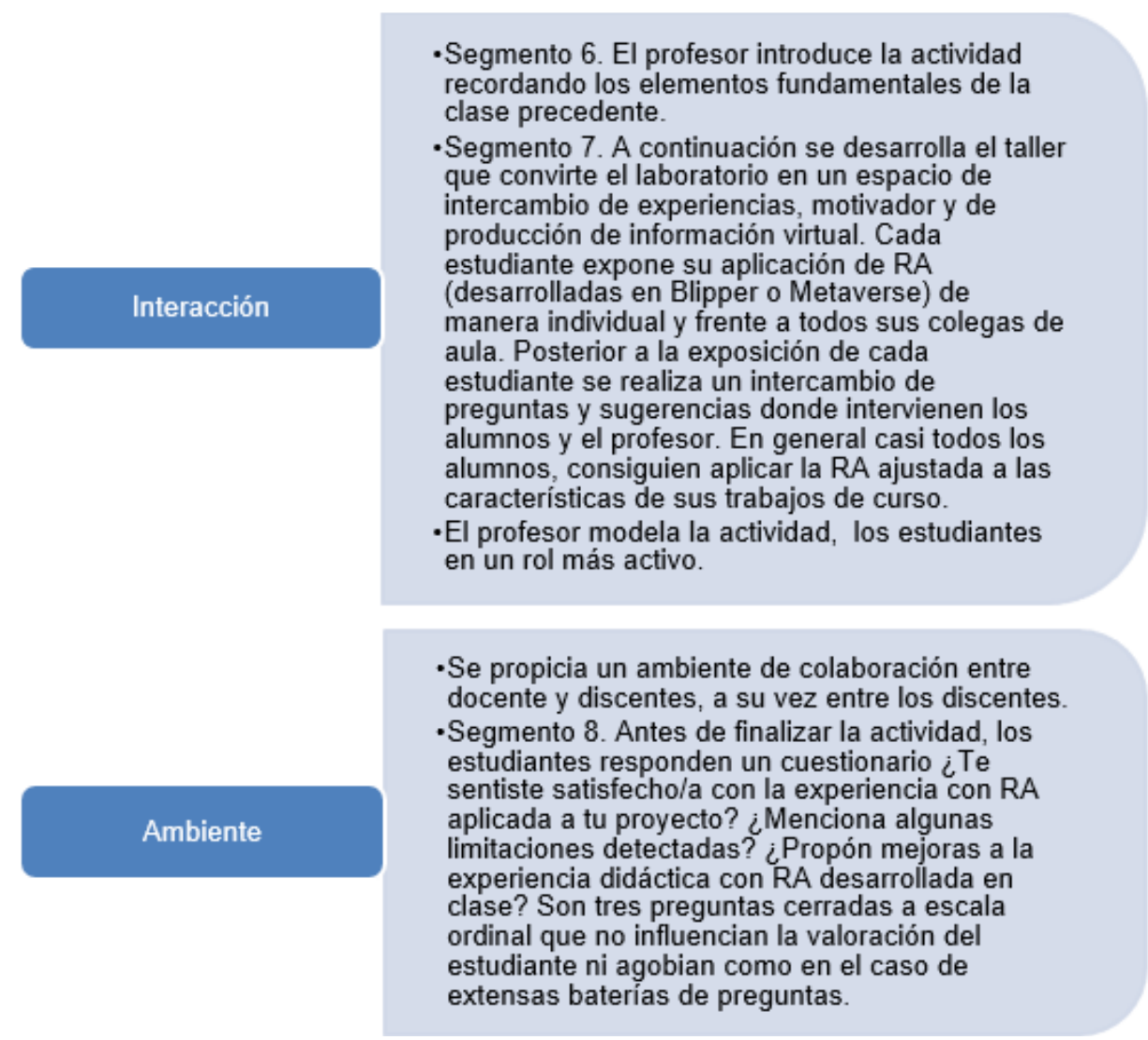

Fuente: elaboración propia utilizando LibreOffice.

\section{Análisis del cuestionario aplicado en la primera clase}

A partir del análisis de los datos recopilados en el cuestionario aplicado en la primera clase, se evidencia que la expectativa de uso de la RA fue de un $22 \%$ alta frente a un $78 \%$ de medias y bajas. La motivación presentó valores aceptables y a la vez alentador para el éxito de la segunda actividad, ver figura 5. 
Figura 5. Gráfico comparativo entre las variables motivación y expectativas de uso de la RA

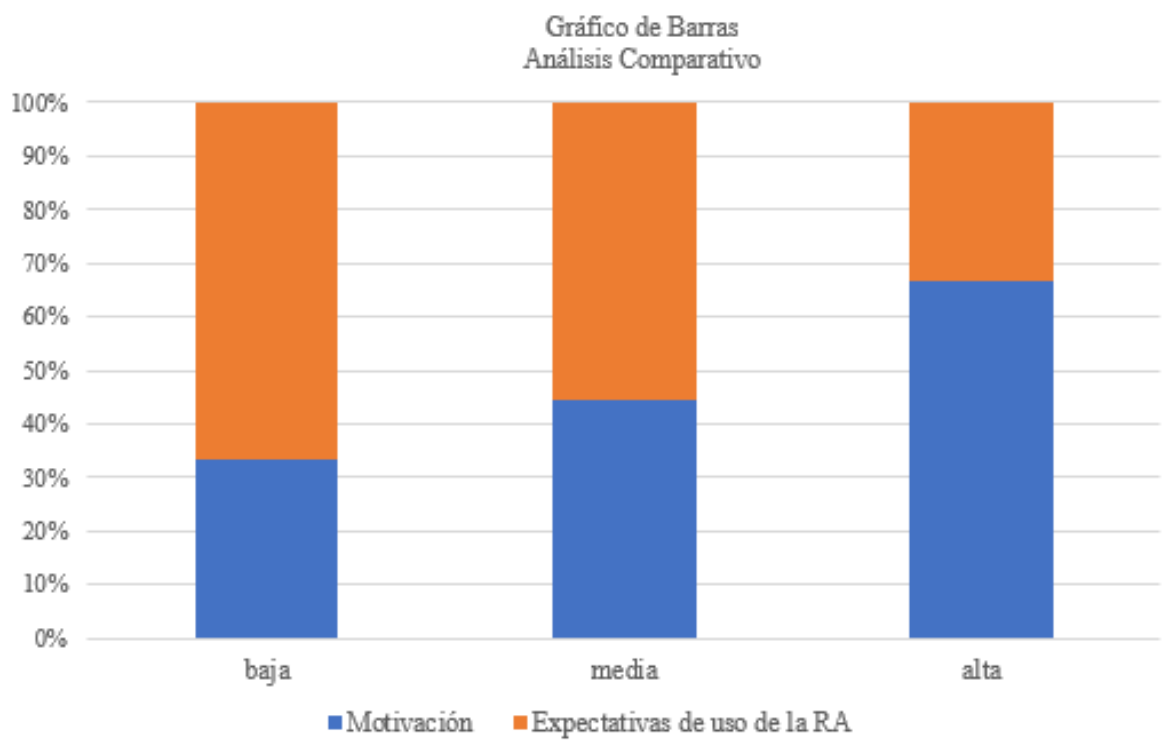

Fuente: elaboración propia utilizando LibreOffice.

\section{Análisis del cuestionario aplicado en la segunda clase}

Los resultados del cuestionario aplicado a los estudiantes en la segunda clase, muestran un $67 \%$ de alta satisfacción al concluir la actividad, mientras que el 22\% de estudiantes se sintió medianamente satisfecho. En la figura 6 se observa el comportamiento de las expectativas de uso de la RA frente a la satisfacción al concluir la actividad (que pudiera ser un indicador del "éxito" de la actividad). 
Figura 6. Análisis comparativo de las variables expectativas de uso de la RA y satisfacción al concluir la actividad

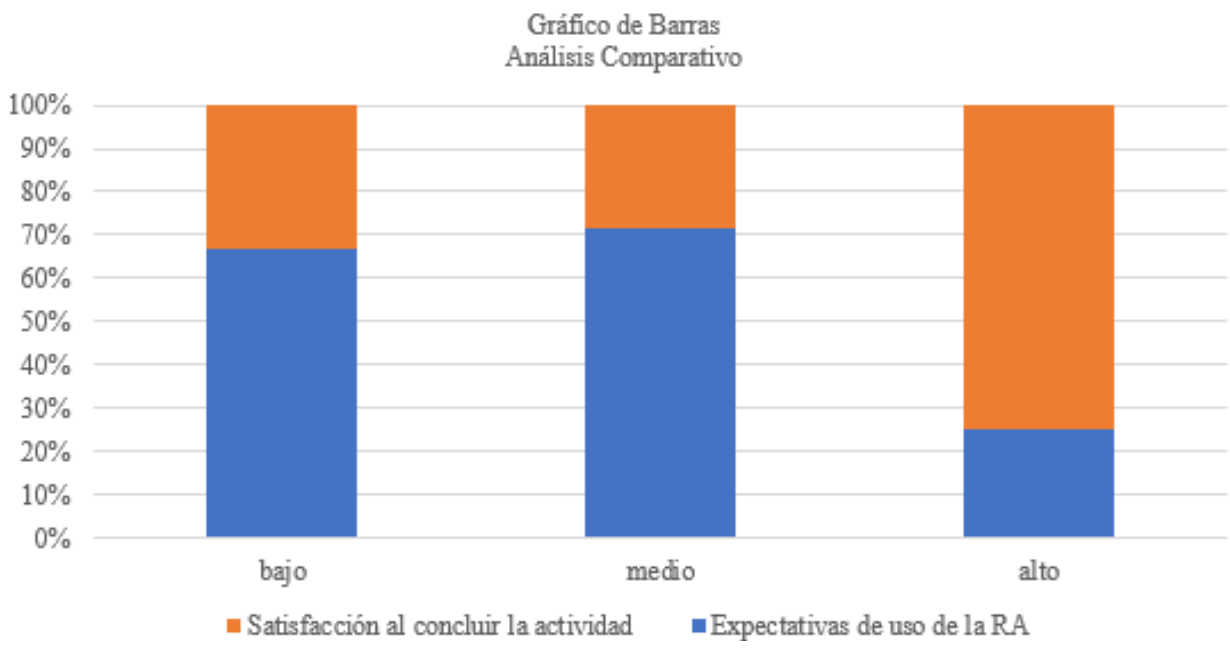

Fuente: Elaboración propia utilizando LibreOffice.

\section{Prueba estadística Chi cuadrado}

El éxito de la tarea en este análisis, es equivalente al logro del estudiante de poder aplicar la RA a su proyecto de curso. A partir del comportamiento descriptivo de las variables medidas podemos inferir que existe relación entre las expectativas de uso de la RA y el éxito de la tarea (ver tabla 1).

Para demostrar este planteamiento se realiza un estudio de inferencia a través de una prueba de hipótesis Chi cuadrado. Siendo la variable principal: expectativas de uso de la RA y variable secundaria: éxito de la tarea. La hipótesis nula Ho: no hay dependencia entre las variables expectativas de uso de la RA y el éxito de la tarea y la hipótesis alternativa H1: hay dependencia entre expectativas de uso de la RA y el éxito de la tarea.

Tabla 1. Tabla de frecuencias

\begin{tabular}{|l|l|l|l|l|l|}
\hline \multicolumn{1}{|c|}{ Expectativa de uso de la RA } & \multicolumn{5}{c|}{ Éxito de la tarea } \\
\hline Bajo & Bajo & \multicolumn{2}{c|}{ Medio } & \multicolumn{1}{c|}{ Alto } & \multicolumn{1}{c|}{ Totales } \\
\hline Medio & 0 & 1 & 1 & 2 \\
\hline Alto & 1 & 1 & 1 & 3 \\
\hline Totales & 0 & o & 4 & 4 \\
\hline
\end{tabular}

Fuente: Elaboración propia utilizando LibreOffice. 
La hipótesis nula de ausencia de relación entre expectativas de uso de la RA y éxito de la tarea, no se puede rechazar (fijando una significación del 5\%; el contraste Chi-cuadrado proporciona un p-valor de 0.287), si bien cabe destacar el reducido número de datos, ver tabla 2.

Tabla 2. Prueba Chi Cuadrado (Fuente: elaboración propia utilizando LibreOffice)

\begin{tabular}{|l|l|}
\hline \multicolumn{2}{|l|}{ Test de independencia (Chi cuadrado) } \\
\hline Alfa & 0,05 \\
\hline Df & 4 \\
\hline Valor P & 0,287 \\
\hline Estadística da proba & 5 \\
\hline Valor crítico & 9,487 \\
\hline
\end{tabular}

Fuente: Elaboración propia utilizando LibreOffice.

\section{Continuación del análisis del cuestionario aplicado en la segunda clase}

Siguiendo el análisis de los resultados obtenidos en el cuestionario aplicado en la segunda clase, se puede concluir que la satisfacción en los estudiantes aumenta en la medida que eran capaces de conseguir el éxito de la tarea (estudiantes 2,3,5,6,7,9). En otros estudiantes que no tuvieron éxito en la realización de la tarea (estudiantes $1,4)$, su satisfacción aumentó cuando percibieron que sus colegas si habían tenido resultados interesantes en la experiencia con RA (ver tabla 3). La tabla 4 refleja la conversión a datos cuantitativos realizada en este estudio.

\section{Ventajas y limitaciones señaladas por los estudiantes}

Los puntos positivos señalados fueron varios, los estudiantes consideraron las herramientas de RA como interesantes, novedosas, y que logran captar la atención tanto de docentes como de estudiantes. Visualizaron en las herramientas de RA múltiples posibilidades de aplicación y viabilidad funcional para sus propios proyectos. La RA puede ser útil para la resolución de conflicto entre estudiantes. Es una tecnología emergente e innovadora, útil y motivadora tanto para los estudiantes como para los profesores, por lo cual constituye un gran valor añadido en el proceso educativo. Los estudiantes señalaron, que a través del uso de la RA se puede acceder a recursos de la Web y crear enlaces relacionados a informaciones relevantes para los proyectos de cursos.

No obstante, los estudiantes también señalaron limitaciones, el 22\% de los estudiantes percibió debilidad para el desarrollo de la tarea por la falta de competencias digitales que poseían; el 21\% experimentó dificultad para aplicar la RA en sus proyectos por falta de ideas; un $36 \%$ planteó la necesidad de más 
tiempo destinado al estudio de las herramientas presentadas y el 21\% consideró las herramientas como poco flexibles.

Tabla 3. Cuestionario de valoración antes, durante y después de la actividad

\begin{tabular}{|c|c|}
\hline \multicolumn{2}{|c|}{$\begin{array}{l}\text { En esta actividad se aplica al finalizar la segunda actividad docente, en la misma los } \\
\text { estudiantes deben valorar la experiencia didáctica con RA en tres momentos diferentes: } \\
\text { antes, durante y después de la actividad. Todo ello, analizando los parámetros de } \\
\text { motivación, expectativas de uso de la RA, satisfacción y mejoras al proceso. }\end{array}$} \\
\hline $\begin{array}{l}\text { Al no haber podido concretar ninguna propuesta de aplicación no podría } \\
\text { decir que me encuentre satisfecho, pero en base a las ideas presentadas por } \\
\text { mis compañeros Sí que le veo una mayor aplicación y viabilidad funcional en } \\
\text { mi propio proyecto. }\end{array}$ & Estudiante 1 \\
\hline $\begin{array}{l}\text { Mis expectativas eran bastante bajas, pero mejoraron después de utilizar } \\
\text { la herramienta ya que observé las múltiples posibilidades que ofrece. } \\
\text { Limitaciones: se necesita estar registrado para poder ver la RA y si no estás } \\
\text { familiarizado con el APP es bastante complicado para editar y publicar. } \\
\text { Sugiero que el APP podría incorporar mejoras que la hiciera más intuitiva. }\end{array}$ & Estudiante 2 \\
\hline $\begin{array}{l}\text { Estoy realmente satisfecho con la experiencia de RA, ya que conseguí ver una } \\
\text { aplicación útil en mi proyecto. Concretamente aplique la RA a la resolución } \\
\text { de conflicto entre estudiantes, utilizando la APP de Blippar. Las limitaciones } \\
\text { que encontré fueron con la familiarización de la herramienta y el tiempo } \\
\text { empleado. }\end{array}$ & Estudiante 3 \\
\hline $\begin{array}{l}\text { Fue estresante porque no vi relación con mi proyecto, aunque creo que la RA } \\
\text { es innovadora, útil y motivadora para los estudiantes. }\end{array}$ & Estudiante 4 \\
\hline $\begin{array}{l}\text { Al principio no veía claro la posibilidad de aplicación de la RA en mi } \\
\text { proyecto. Tras investigar sobre el Blippar he encontrado la forma de } \\
\text { usar esta aplicación. La principal limitación considero que fue el tiempo } \\
\text { destinado a la actividad, ya que me hubiera gustado más tiempo para ser más } \\
\text { productivo y potenciar este recurso. }\end{array}$ & Estudiante 5 \\
\hline $\begin{array}{l}\text { Creo que la RA es un gran valor añadido, al principio no sabía cómo aplicarla } \\
\text { en mi proyecto. Me he sentido satisfecha porque he logrado crear algo. El } \\
\text { idioma fue una limitación, la herramienta poco es flexible y hay poco uso de } \\
\text { videos explicativos. }\end{array}$ & Estudiante 6 \\
\hline $\begin{array}{l}\text { Creo que la RA es más que un instrumento de motivación, sin embargo, al } \\
\text { principio no tenía claro cómo aplicarla en mi proyecto. A lo largo de la clase } \\
\text { decidí que voy a utilizar la RA para acceder a recursos de la Web Compostela } \\
\text { y crear enlaces relacionados a informaciones relevantes de mi proyecto. } \\
\text { Me siento satisfecha con la experiencia, la considero interesante. Aunque } \\
\text { propongo que se debería disponer de más tiempo para llevarla a cabo. }\end{array}$ & Estudiante 7 \\
\hline $\begin{array}{l}\text { Al principio me sentí motivada con la propuesta de aplicar la RA en mi } \\
\text { proyecto, pero después me sentí perdida a la hora de aplicarla en mi } \\
\text { proyecto. Las limitaciones que tuve fueron la falta de ideas y propongo más } \\
\text { tiempo destinado a experiencias de este tipo. }\end{array}$ & Estudiante 8 \\
\hline
\end{tabular}


La RA me parece una herramienta interesante y novedosa que logra captar la atención tanto de docentes como de estudiantes. Por lo pronto no veo más limitaciones que las mías propias en el desempeño de la actividad y todo lo relacionado con las TIC.

Fuente: Elaboración propia utilizando LibreOffice.

Tabla 4. Conversión a datos cuantitativo realizada en este estudio

\begin{tabular}{|c|c|c|c|c|c|c|c|c|c|c|}
\hline & \multicolumn{3}{|c|}{ Motivación } & \multicolumn{3}{|c|}{$\begin{array}{c}\text { Expectativas de } \\
\text { uso }\end{array}$} & \multirow{2}{*}{$\begin{array}{l}\text { Éxito } \\
\text { de la } \\
\text { tarea }\end{array}$} & \multicolumn{3}{|c|}{ Satisfacción } \\
\hline & baja & media & alta & baja & media & alta & & baja & media & alta \\
\hline Estudiante 1 & 1 & 0 & o & $\mathrm{O}$ & 1 & o & o & o & 1 & 0 \\
\hline Estudiante 2 & 0 & 1 & o & 1 & 0 & o & 1 & o & o & 1 \\
\hline Estudiante 3 & 0 & 0 & 1 & o & o & 1 & 1 & 0 & 0 & 1 \\
\hline Estudiante 4 & o & 1 & o & o & 1 & 0 & O & 0 & 1 & $\mathrm{O}$ \\
\hline Estudiante 5 & 0 & 0 & 1 & o & 1 & 0 & 1 & 0 & 0 & 1 \\
\hline Estudiante 6 & 0 & o & 1 & 0 & 0 & 1 & 1 & 0 & o & 1 \\
\hline Estudiante 7 & $\mathrm{O}$ & 1 & o & $\mathrm{O}$ & 1 & o & 1 & $\mathrm{O}$ & 0 & 1 \\
\hline Estudiante 8 & 0 & 1 & 0 & 1 & 0 & 0 & 0 & 1 & 0 & 0 \\
\hline Estudiante 9 & o & 0 & 1 & 0 & 1 & $\mathrm{O}$ & 1 & $\mathrm{O}$ & o & 1 \\
\hline Total & 1 & 4 & 4 & 2 & 5 & 2 & 6 & 1 & 2 & 6 \\
\hline
\end{tabular}

Fuente: Elaboración propia utilizando LibreOffice.

\section{DISCUSIÓN}

La integración de las TIC en el sistema educativo contemporáneo es una realidad, las tecnologías se colocan en una dimensión superior como único recurso que permite la continuidad del curso escolar de manera online y en este contexto surgen nuevas competencias digitales (San Nicolás, Vargas y Moreira, 2012; Castañeda Quintero y Adell, 2013, Fernández, Fernández y Cebreiro, 2016). Las instituciones educativas deben poner en marcha nuevos métodos y estilos de aprendizaje que aprovechen al máximo los recursos materiales, tecnológicos y humanos existentes (Fainholc, 2008). En este escenario, es necesario aumentar las investigaciones científicas y mejorar las políticas de gobiernos que garanticen armonía entre equidad e innovación en la Educación (Montoya, 2009; García Aretio, 2017; Vila Merino, 2012).

De la misma manera, las carencias formativas en nuevas competencias digitales que se demandan en la sociedad actual deben ser identificadas, es necesario una "alfabetización tecnológica" para formar estudiantes críticos en tecnologías avanzadas como requisito indispensable para el desarrollo profesional de la nueva generación (Marzal, 2008; Palomeque-Córdova, 2020; Aguaded Gómez, Marín Gutiérrez y Díaz Pareja, 2015). Las modificaciones curriculares con el fin de incorporar formación 
en tecnologías avanzadas deben ser una prioridad (Labra, 2010) de esta forma trasciende el carácter instrumental de los medios y se logra integrar el conocimiento tecnológico avanzado con el conocimiento didáctico de su utilización (Ramírez García y González Fernández, 2016).

Por otro lado, el uso de dispositivos móviles inteligentes adquiere gran importancia en la educación, estos pueden contribuir a disminuir la brecha digital debido a la reducción de costes en equipamientos electrónicos y lo versátil que resultan estos dispositivos (Barroso Osuna y Cabero Almenara, 2016; Cabero Almenara, Fernández Robles y Marín Díaz, 2017). Las aplicaciones de la RA que permiten al usuario ver el mundo real con objetos virtuales superpuestos o compuestos con el mundo real, a través de una pantalla de móvil convencional (Bogen, Wind y Giuliano, 2006; Noh, Sunar y Pan, 2009). En la educación, la RA permite a los estudiantes adquirir habilidades para el acceso, análisis, evaluación y producción creativa de nuevos conocimientos (Arribas, Gutiérrez, Gil y Santos, 2014). La RA tiene muchas posibilidades de aplicación en el terreno educativo, ayuda a aumentar la motivación en los estudiantes, contribuye a disminuir el tiempo necesario para comprender temas complejos, permite recrear ambientes difíciles de reproducir y permite la interacción entre objetos y recursos de diversas naturalezas (Wu, Lee, Chang y Liang, 2013). Algunos ejemplos de aplicación a varios dominios de aprendizaje fueron presentados en este trabajo como museos de juguetes, área de arqueología y restauración, juegos virtuales, tele-robótica y aplicación en el área de la ingeniería (Sarracino, 2014; Usó y García, 2014; Noh, Sunar y Pan, 2009; Marín, Sanz y Del Pobil, 2003; Toguchi, Sasaki, Mizuno y Shikoda, 2011).

El diseño de la experiencia didáctica con RA, promueve acciones formativas que contribuyen a la preparación del estudiante en el diseño, la producción y la utilización de los recursos virtuales con tecnología de RA. La deconstrucción de las clases supuso el análisis de información recolectada en dos niveles: descriptivo e interpretativo, para ello se segmenta la información recopilada, teniendo en cuenta diferentes fases: espacio-tiempo, contenido-método, discursos, interacción y ambiente (Stodolsky, 1991). Este estudio propició el compromiso de docentesdiscentes para seguir trabajando en las prioridades que la educación actual exige, una formación sólida y la adquisición de nuevas competencias digitales. Los estudiantes se sintieron satisfechos al concluir la actividad, señalaron ventajas y limitaciones lo que contribuye al perfeccionamiento de nuevas experiencias futuras en otros dominios de aplicación (Candioti y Medina, 2007).

Para finalizar, el estudio piloto permite visualizar a los estudiantes en un nuevo rol como prosumidores de contenidos virtuales, lo que constituye una nueva competencia digital en los futuros profesionales de la educación, antes reservada a desarrolladores informáticos. 


\section{REFERENCIAS}

Aguaded Gómez, J. I., Marín Gutiérrez, I., \& Díaz Pareja, E. M. (2015). La alfabetización mediática entre estudiantes de primaria y secundaria en Andalucía (España). RIED: Revista Iberoamericana de Educación a Distancia, 18(2), 275-298. https://doi. org/10.5944/ried.18.2.13407

Arribas, J. C., Gutiérrez, S. M., Gil, M. C., y Santos, A. C. (2014). Recursos digitales autónomos mediante realidad aumentada. RIED. Revista Iberoamericana de Educación a Distancia, 17(2), 241-274. https://doi.org/10.5944/ried.17.2.12686

Azuma, R. (1997). A Survey of Augmented Reality. Presence Teleoperators and Virtual Environments, 6(4), 355-385. https://doi.org/10.1162/ pres.1997.6.4.355

Barroso Osuna, J. M., y Cabero Almenara, J. (2016). Evaluación de objetos de aprendizaje en realidad aumentada: estudio piloto en el grado de Medicina. Enseñanza \& Teaching, 34(2), 149-167. http://hdl.handle.net/11441/51387

Bogen, M., Wind, J., y Giuliano, A. (2006, October). ARISE-Augmented Reality in school environments. In European Conference on Technology Enhanced Learning (pp. 709-714). Springer, Berlin, Heidelberg. https://doi. org/10.1007/11876663 77

Cabero Almenara, J., Fernández Robles, B., y Marín Díaz, V. (2017). Dispositivos móviles y realidad aumentada en el aprendizaje del alumnado universitario. RIED. Revista Iberoamericana de Educación a Distancia, 2o(2), 167-185. https://doi.org/10.5944/ried.20.2.17245

Cabero Almenara, J., y García, F. (2016). Realidad aumentada. Tecnología para la formación. Madrid: Síntesis [Reseñas]. Pixel-Bit: Revista de Medios y Educación, 49, 241-242. https://recyt.fecyt.es/index. $\mathrm{php} / \mathrm{pixel} /$ article/view/61727
Castañeda Quintero, L. J., y Adell, J. (2013). Entornos Personales de Aprendizaje: claves para el ecosistema educativo en red. Editorial Marfil. http://www.um.es/ ple/libro/

Deshpande, S., Kank, T., Armanyous, M., Singh, S., y Kalita, M. (2020). Improvised learning for pre-primary students using augmented reality. TechRxiv. Preprint. I: https://doi.org/10.36227/ techrxiv.12056046.v1

Dunleavy, M., Dede, C., y Mitchell, R. (2009). Affordances and limitations of immersive participatory augmented reality simulations for teaching and learning. Journal of science Education and Technology, 18(1), 7-22. https:// www.jstor.org/stable/23036161. https:// doi.org/10.1007/s10956-008-9119-1

De la Horra Villacé, I. (2017). Realidad aumentada, una revolución educativa. Edmetic, 6(1), 9-22. https://doi. org/10.21071/edmetic.v6i1.5762

El-Hakim, S. F., Beraldin, J. A., Picard, M., y Godin, G. (2004). Detailed 3D reconstruction of large-scale heritage sites with integrated techniques. IEEE computer graphics and applications, 24(3), 21-29. https://doi. org/10.1260/147807709788549439

Escartín, E. R. (2000). La realidad virtual, una tecnología educativa a nuestro alcance. Pixel-Bit. Revista de Medios y Educación, 15, 5-21. http://hdl.handle. net/11441/45510

Fainholc, B. (2008). De cómo las TICs podrían colaborar en la innovación sociotecnológico-educativa en la formación superior y universitaria presencial. RIED. Revista iberoamericana de educación a distancia, 11(1), 53-79. https://doi. org/10.5944/ried.1.11.956

Fernández de la Iglesia, J. C., Fernández Morante, M. C., y Cebreiro López, B. (2016). Desarrollo de un cuestionario 
de competencias en TIC para profesores de distintos niveles educativos. PixelBit: Revista de Medios y Educación, 48, 135-148. https://doi.org/10.12795/ pixelbit.2016.i48.09

García Aretio, L. (2017). Educación a distancia y virtual: calidad, disrupción, aprendizajes adaptativo y móvil. RIED. Revista Iberoamericana de Educación a Distancia, 2O(2), 9-25. https://doi. org/10.5944/ried.20.2.18737

García-Ruiz, R., Aguaded, I., y Bartolomé, A. R. (2017). La revolución del 'blended learning' en la educación a distancia. RIED. Revista Iberoamericana de Educación a Distancia, 2(1), 25-32. https://doi.org/10.5944/ried.21.1.19803

Klopfer, E. (2008). Augmented learning: Research and design of mobile educational games. MIT press. https:// www.jstor.org/stable/40607096? seq=1. https://doi.org/10.7551/ mitpress/9780262113151.001.0001

Klopfer, E., y Squire, K. (2008). Environmental Detectives-the development of an augmented reality platform for environmental simulations. Educational technology research and development, 56(2), 203-228. https:// doi.org/10.1007/s11423-007-9037-6

Johnson, L., Levine, A., Smith, R., y Stone, S. (2010). Simple augmented reality. The 2010 Horizon Report, 1. https://eric. ed.gov/?id=ED510220

Labra, J. P. (2010). Tecnología educativa. La formación del profesorado en la era de Internet. Archidona, Aljibe. Revista Fuentes, 10, 222-225. http://hdl.handle. net $/ 11441 / 32884$

Marín-Díaz, V., y Sampedro-Requena, B. E. (2020). La Realidad Aumentada en Educación Primaria desde la visión de los estudiantes. Alteridad. Revista de Educación, 15(1), 61-73. http://dx.doi. org/10.17163/alt.v15n1.2020.05

Marín, R., Sanz, P. J., y Del Pobil, A. P. (2003). The UJI online robot: An education and training experience. Autonomous Robots, 15(3), 283-297. https://doi. org/10.1023/A:1026220621431

Marshall, C., \& Rossman, G. B. (2014). Designing qualitative research. Sage publications. https://escholarship.org/ uc/item/3m25g8j8

Marzal, M. Á. (2008). La alfabetización en información como dimensión de un nuevo modelo educativo: la innovación docente desde la documentación y los CRAI. RIED. Revista iberoamericana de educación a distancia, 11(2), 41-66. https://doi.org/10.5944/ried.2.11.930

Milgram, P., Takemura, H., Utsumi, A., y Kishino, F. (1995, December). Augmented reality: A class of displays on the realityvirtuality continuum. In Telemanipulator and telepresence technologies, 2351, (282-292). International Society for Optics and Photonics. https://doi. org/10.1117/12.197321

Montoya, M. S. R. (2009). Recursos tecnológicos para el aprendizaje móvil (mlearning) y su relación con los ambientes de educación a distancia: implementaciones e investigaciones. RIED. Revista iberoamericana de educación a distancia, 12(2), 57-82. http://e-spacio.uned.es/fez/view/ bibliuned:revistaRied-2009-12-2-2040

Noh, Z., Sunar, M. S., y Pan, Z. (2009, August). A review on augmented reality for virtual heritage system. In International conference on technologies for E-learning and digital entertainment (pp. 50-61). https://doi.org/10.1007/9783-642-03364-3 7

Palomeque-Córdova, I. D. T. (2020). Córdova, I. P. (2020). Programa de alfabetización tecnológica para los estudiantes universitarios. Polo del Conocimiento: Revista científicoprofesional, 5(1), 477-499. doi: 10.23857/ pc.v5io1.1234

Preissle, J. (2008). How to be an inspired qualitative methodologist: learning from 
Egon Guba and his work. https://doi. org/10.1080/09518390802489014

Ramírez García, A., y González Fernández, N. (2016). Competencia mediática del profesorado y del alumnado de educación obligatoria en España. Revista Científica de Educomunicación, Comunicar, 49, 4958. https://doi.org/10.3916/C49-201605

San Nicolás, M. B., Vargas, E. F., y Moreira, M. A. (2012). Competencias digitales del profesorado y alumnado en el desarrollo de la docencia virtual. El caso de la Universidad de La Laguna. Revista Historia de la Educación Latinoamericana, 14(19). https://doi. org/10.9757/Rhela.19.10

Sarracino, F. (2014). ¿Mejora la Realidad Aumentada el aprendizaje de los alumnos? Una propuesta de experiencia de museo aumentado. Revista Profesorado, 18(3), 473,491. $\quad$ http://hdl.handle. net $/ 10481 / 34531$

Stodolsky, S. S. (1991). La importancia del contenido en la enseñanza: Actividades en las clases de matemáticas y ciencias sociales. Madrid: Paidós.

Toguchi, A., Sasaki, H., Mizuno, K., y Shikoda, A. (2011). Build a prototype of new e-Learning contents by using the AR technology. In Engineering and
Information Systems, Technologies and Applications 2011, The 5th International Multi-Conference on Society (pp. 261264). https://api.semanticscholar.org/ CorpusID:164215686

Usó, J. M., y García, A. C. (2014). Aplicación de las nuevas tecnologías para la difusión didáctica del patrimonio. Tejuelo: Didáctica de la Lengua y la Literatura. Educación, 9, 779-788. https://dialnet.unirioja.es/servlet/ articulo?codigo $=5385972$

Van Teijlingen, E., y Hundley, V. (2002). The importance of pilot studies. Nursing Standard 16(40), 33. 10.7748/ ns2002.06.16.40.33.c3214

Vila Merino, E. S. (2012). Ciudadanía, equidad e innovación: reflexiones sobre la política de responsabilidad social de las universidades. Innovación educativa (México, DF), 12(59), 61-85. http://www. scielo.org. $\mathrm{mx} /$ scielo.php?pid $=\mathrm{S} 1665-$ $26732012000200006 \& \mathrm{script}=\mathrm{sci}$ abstract

Wu, H. K., Lee, S. W. Y., Chang, H. Y., y Liang, J. C. (2013). Current status, opportunities and challenges of augmented reality in education. Computers \& Education, 62, 41-49. https://doi.org/10.1016/j. compedu.2012.10.024

\section{PERFIL ACADÉMICO Y PROFESIONAL DE LAS AUTORAS}

Inés María González Vidal. Miembro del Grupo de Investigación en Tecnología Educativa (Tecnoeduc). Líneas investigación e-learning, hipermedia adaptativa, sistemas de información. Fue profesora de la Facultad de Contabilidad y Finanzas, Universidad de la Habana y profesora sustituta del Instituto de Computación, Universidad Federal de Alagoas. Actualmente vinculada al programa de Equidad e Innovación de la Facultad de Educación de la Universidad de Santiago de Compostela. https://orcid.org/0000-0003-0559-0321

E-mail: inesmaria.gonzalez@rai.usc.es 
Beatriz Cebreiro López. Directora del Grupo de Investigación Tecnología Educativa (Tecnoeduc) con código GI-1438. Experta en Tecnología Educativa. Líneas investigación e-learning, innovación con TIC. Fue directora del Campus Virtual y del Plan de Formación del Profesorado Universitario de la Universidad de Santiago de Compostela. Miembro de la Asociación Internacional para el desarrollo la Tecnología Educativa y de las Nuevas Tecnologías aplicadas a la educación (Edutec) y del observatorio gallego de e-learning. https://orcid.org/00oo-0003-2064-915X E-mail: beatriz.cebreiro@usc.es

Lorena Casal Otero. Miembro del Grupo de Investigación en Tecnología Educativa de la Universidad de Santiago de Compostela. Hasta el año 2018, técnico e-Learning en el Centro de Tecnologías de Aprendizaje de la USC, responsable de la gestión del Campus Virtual de la Universidad. Docente en diferentes actividades formativas relacionadas con uso didáctico de las TIC realizadas a través del Plan de Formación en Innovación Docente de la USC. https://orcid.org/0000-0002-09064321

E-mail: lorena.casal@usc.es

Dirección:

Facultad de Ciencias de la Educación

Campus Vida: Rúa Prof. Vicente Fráiz Andón, s/n.

Universidade de Santiago de Compostela

Fecha de recepción del artículo: 12/05/2020

Fecha de aceptación del artículo: 03/07/2020

Fecha de aprobación para maquetación: 13/08/2020 